Cahiers $d u$ MONDE RUSSE

\section{Cahiers du monde russe}

Russie - Empire russe - Union soviétique et États indépendants

$50 / 4 \mid 2009$

Varia

\title{
Michael Geyer, Sheila Fitzpatrick, eds., Beyond Totalitarianism
}

\section{Alain Blum}

\section{OpenEdition}

\section{Journals}

Édition électronique

URL : https://journals.openedition.org/monderusse/7252

DOI : 10.4000/monderusse. 7252

ISSN : $1777-5388$

\section{Éditeur}

Éditions de l'EHESS

\section{Édition imprimée}

Date de publication : 15 décembre 2009

Pagination : 935-939

ISBN : 978-2-7132-2261-0

ISSN : $1252-6576$

Référence électronique

Alain Blum, "Michael Geyer, Sheila Fitzpatrick, eds., Beyond Totalitarianism », Cahiers du monde russe [En ligne], 50/4 | 2009, mis en ligne le 12 janvier 2011, consulté le 03 septembre 2022. URL : http:// journals.openedition.org/monderusse/7252; DOI : https://doi.org/10.4000/monderusse.7252

Ce document a été généré automatiquement le 3 septembre 2022

Tous droits réservés 


\title{
Michael Geyer, Sheila Fitzpatrick, eds., Beyond Totalitarianism
}

\author{
Alain Blum
}

\section{RÉFÉRENCE}

Michael GEYER, Sheila FITZPATRICK, eds., Beyond Totalitarianism. Stalinism and Nazism

Compared. Cambridge, MA : Cambridge University Press, 2009, 536 p.

1 La comparaison entre régime stalinien et régime nazi constitue sans doute l'un des objets d'étude les plus courants comme les plus polémiques. Depuis l'émergence du concept de totalitarisme au fondement de la comparaison entre les deux régimes jusqu'à aujourd'hui, les réflexions sur la possibilité d'établir un parallèle entre ces deux systèmes reviennent régulièrement au centre de la scène scientifique. Rappelons, parmi les ouvrages récents sur la question, Stalinisme et nazisme. Histoire et mémoires comparées ${ }^{1}$, qui mettait en parallèle plusieurs contributions de Nicolas Werth et Philippe Burrin, ou encore le « dialogue » entre Ian Kershaw et Moshe Lewin dans Stalinism and nazism: Dictatorship in comparison ${ }^{2}$.

2 Cet ouvrage, Beyond Totalitarianism, élaboré sous la direction de Michael Geyer et Sheila Fitzpatrick, l'un spécialiste de l'histoire de l'Allemagne nazie, l'autre de celle de l'URSS stalinienne, et qui rassemble des contributions signées des meilleurs historiens, innove cependant par de nombreux aspects. Il ouvre une perspective féconde poursuivant une démarche ostensiblement comparatiste. L'objectif est posé dans une introduction rédigée à deux voix : se dégager du cadre par trop simplificateur et surtout contraignant $\mathrm{du}$ totalitarisme pris comme un tout, pour engager des comparaisons: «Our enterprise started with no shared, a priori position on the value of this comparison or on the balance of similarities and differences between Stalinism and Nazism » (p. 27). Les deux éditeurs délimitent ainsi clairement la période de l'étude - années 1930 et 1940 - afin de mettre en lumière « the quintessential features of Nazism and Stalinism », 
renonçant dès lors à réfléchir sur les racines de ces expériences comme sur leurs conséquences.

Le livre est organisé en une série de chapitres co-écrits par deux auteurs : tel est l'aspect le plus original du projet, mené lors de séminaires organisés par Geyer et Fitzpatrick, au cours desquels les futurs contributeurs discutaient afin d'élaborer ces pages écrites à quatre mains. S'y alternent, dans un équilibre parfait, des analyses des deux systèmes, conduisant à des conclusions focalisées sur la comparaison. Chaque chapitre traite d'un aspect particulier de la violence nazie et stalinienne, puisque c'est cette donnée qui structure volontairement l'interrogation ("the emphasis on the intensity and aggressiveness of action that characterized the peculiar historicity of the two regimes suggested that we study practice »). L'idéologie n'est jamais au cœur de l'analyse, même si elle est évidemment présente à de multiples reprises, dès que sont traitées par exemple, intégration dans la société ou exclusion, création d'un homme nouveau ou représentations de l'autre.

4 Quatre grandes parties structurent l'ensemble: "Gouvernance », "Violence », "Socialisation", et enfin « Enchevêtrements " (Entanglements), dernière section qui traite de l'interaction entre les deux systèmes.

5 La gouvernance $\left(1^{\text {re }}\right.$ p.) est au cœur de la réflexion sur le totalitarisme. Elle est analysée ici par Yoram Gorlizki et Hans Mommsen, qui traitent de la nature de l'autocratie des deux régimes, puis par David Hoffmann et Annette Timm, qui étudient une dimension particulière de la gouvernance, s'ancrant dans la sphère de la biopolitique. Gorlizki et Mommsen sont particulièrement attentifs à la cohérence des deux "machines", soulignant la très forte intégration propre au système soviétique, qui explique selon eux la remarquable stabilité de ce dernier malgré le choc de la Seconde Guerre mondiale. Stalin est obsédé par la construction de nouvelles formes étatiques, et c'est bien cette intégration de la société à l'État-parti qui est au cœur de son action. À l'inverse, Hitler ne porte guère d'intérêt à la construction d'un État propre à sa domination et en rupture avec la période précédente; il impose par ailleurs des séparations strictes entre les différentes sphères de gouvernance, ce qui empêche de parler d'État nazi. Les formes de mobilisation sont dès lors profondément différentes, le système stalinien reposant bien davantage sur ces formes d'intégration, alors que le régime hitlérien est fondé sur l'agitation et la propagande. Le deuxième chapitre (D. Hoffmann et A. Timm) apparaît sans doute moins important dans cet exercice comparatif et il ne semble pas certain que la biopolitique soit indispensable comme clé de comparaison. Car il n'y a pas, derrière les deux systèmes, de marque spécifique d'une forme de biopolitique, mais bien la dominance d'une idéologie propre, l'une raciale, qui conduit l'essentiel de la démarche biopolitique de l'Allemagne nazie, l'autre guidée, dans l'URSS stalinienne, par une démarche moins étrangère à ce qui se fait ailleurs en Europe et dans laquelle l'obsession du développement de la force de travail est centrale.

6 La deuxième partie, consacrée aux formes de la violence, est évidemment essentielle dans une comparaison des deux systèmes les plus brutaux et meurtriers du $\mathrm{xx}^{\mathrm{e}}$ siècle. Christian Gerlach et Nicolas Werth engagent ainsi un dialogue passionnant sur la violence de masse. Ils soulignent d'abord les points communs: une tendance à la professionnalisation de la violence, mise en œuvre par des organes étatiques développés dans ce seul but. Si certaines périodes sont marquées par une instrumentalisation de la violence d'en-bas - et surtout si la violence, dans les deux cas, est connue d'une large part de la population qui en bénéficie parfois -, elle n'en est pas moins professionnalisée, 
organisée par l'État et perpétrée par des personnels spécialisés dans la répression. Ces points de convergence entre l'Allemagne nazie et l'URSS stalinienne sont opposés à ce qui, aux yeux des deux historiens, offre la plus grande divergence entre les deux systèmes. Une violence extrême dans l'Allemagne nazie, fondée sur des plans qui ne seront pas réalisés jusqu'au bout et sont majoritairement orientés vers des populations extérieures à l'Allemagne; soit une violence dite «impérialiste». Une violence, dans l'URSS stalinienne, reposant sur des plans qui seront souvent dépassés suite aux modalités de leur mise en pratique et aux initiatives locales, violence par ailleurs essentiellement dirigée vers des ennemis intérieurs.

7 La vision de la différence et de son éradication constitue un point commun de la représentation des populations, dans l'Allemagne nazie comme dans l'URSS stalinienne. La place prépondérante que tiennent les nomenclatures et les classifications, produites en particulier par les milieux académiques, est essentielle pour comprendre les politiques des deux États envers les populations vivant ou non sur leurs territoires (Jörg Baberowski et Anselm Doering-Manteuffel). Des différences fondamentales rendent peu commensurables les pratiques issues de ces représentations, même si ces pratiques aboutirent dans les deux cas à des formes d'épuration ethnique. À nouveau, si la violence de l'Allemagne nazie est plus orientée vers l'extérieur et tend à son paroxysme à partir de l'invasion de la Pologne, la terreur stalinienne est orientée vers l'intérieur. Mais, plus encore, la distinction réside dans la spécificité de cette volonté de suppression des différences, qui passe par l'extermination dans l'Allemagne nazie et par la transformation dans l'URSS stalinienne, même si la déportation en est un des outils.

8 Les populations sont ainsi classifiées et les politiques menées en fonction de ces catégorisations ( $3^{\mathrm{e}} \mathrm{p}$.: "Socialisation»). Cependant, celles-ci ne signifient pas la disparition de tout lien transversal, bien au contraire. Sheila Fitzpatrick et Alf Lüdtke soulignent la multiplicité des formes de relations transversales à travers la création de collectifs en URSS (les komsomols par exemple) ou l'usage d'associations en Allemagne, conduisant au développement de diverses formes de sociabilité. L'adhésion au projet politique de l'État repose sur une claire distinction entre inclusion de ceux qui développent un enthousiasme réel, en particulier les jeunes, regroupés dans des formes particulières d'associations, et exclusion sur des bases raciales en Allemagne nazie, sociales en Union soviétique. Une société se développe, dans l'Allemagne hitlérienne comme en URSS, sur de nouvelles formes de liens : " "Mass society" in general , and "totalitarian rule" in particular, may have twisted, devalued, or even destroyed some social relationships - for example, those of kin, class, or milieu - as Hannah Arendt has argued. Concomitantly, though, in both societies people generated new relationships and transformed old ones. » Focalisation sur les jeunes, mobilisation, inclusion et exclusion caractérisent ainsi ces deux sociétés. Fitzpatrick et Lüdtke placent, au fondement de cette transformation du quotidien, ce qu'ils nomment energizing paradigm - un engagement massif fondé sur une forte adhésion et des périodes d'enthousiasme et d'activité extrêmes.

9 Peter Firtzsche et Jochen Hellbeck se placent dans cette perspective tout en l'élargissant. Ils s'interrogent sur l'homme nouveau, tel qu'il est souhaité par la Russie stalinienne et l'Allemagne nazie. Le concept d'homme nouveau n'est évidemment pas né de ces deux régimes et ce chapitre en reconstitue les racines pour souligner que Russie et Allemagne le concrétisent dans deux projets radicalement différents. En Russie, qu'elle soit d'ailleurs prérévolutionnaire, bolchevique ou stalinienne, la transformation de l'esprit 
est essentielle, alors que dans l'Allemagne nazie, on vise plutôt la transformation du corps marquée par l'aryanisation. Stalinisme et nazisme ont cependant en commun « [a] commitment to discipline, whether intellectual or physical, and thus a belief in the ability to leave behind the liberal world ». L'énergie destructrice qui tend à construire la domination de l'homme nouveau mène à croire, dans l'URSS stalinienne, que l'histoire abandonne inéluctablement l'homme ancien, alors qu'en Allemagne nazie, ce sont tous les périls raciaux qui doivent être éliminés.

La dernière section interroge la relation concrète qui s'établit entre les deux puissances. Dans le premier des deux chapitres, Mark Edele et Michael Geyer traitent du processus de brutalisation extrême qui s'établit durant la Seconde Guerre mondiale, d'un " enchevêtrement » qui va tendre vers une guerre totale opposant ces deux mondes. Les auteurs soulignent qu'il y a encore beaucoup à apprendre sur les perceptions des soldats et de la population civile du côté soviétique, alors qu'il existe une immense littérature traitant de ces questions du côté Allemagne nazie. La transformation du conflit en guerre totale, étape par étape, est marquée avant tout par une haine initiale entre les deux pays, une politique militarisée qui conduit à une guerre sur des fronts autant intérieur qu'extérieur, rompant avec une tradition militaire de guerre essentiellement concentrée sur l'extérieur. Le mécanisme de brutalisation se développe dans un processus d'escalade dans lequel, à la montée illimitée de la violence nazie répond une radicalisation de plus en plus forte de la défense soviétique : «Soldiers on both sides committed extraordinary atrocities and the likelihood of their doing so increased with their sense of impunity and just cause, such as revenge ». C'est donc bien par une interaction permanente, et sur un fondement d'opposition radicale, que s'explique ce phénomène.

Le second chapitre (Katerina Clark et Karl Shlögel) traite des représentations croisées de l'Allemagne nazie et de l'URSS stalinienne. Soulignant que ce ne sont pas les «monumentalismes rivaux» de l'Exposition universelle de 1937 qui les traduisent au mieux, ces auteurs démontrent qu'il faut davantage chercher dans l'usage de clichés et autres stéréotypes, bien antérieurs au stalinisme et au nazisme, mais retournés contre ceux que l'on veut détruire. D'un côté, l'Union soviétique se targue d'une suprématie culturelle, d'autant plus revendiquée que l'Allemagne est présentée comme destructrice de sa grande culture; de l'autre, cette dernière met en avant la domination raciale, prétendant que l'URSS a détruit « l'esprit russe ». Enfin, chacune se présente comme le dernier rempart dans la défense de l'Occident.

La démarche de cet ouvrage est à la fois originale et passionnante, faisant apparaître la comparaison dans toute sa complexité. Elle conduit, nous venons de le voir, à d'importantes conclusions et offre à étudier de nouvelles hypothèses. Il s'agit certainement d'une étape nécessaire, voire indispensable, et menée avec conviction, de cette nouvelle écriture de l'histoire des régimes autoritaires et répressifs $\mathrm{du} \mathrm{xx}^{\mathrm{e}}$ siècle. Étape d'autant plus nécessaire qu'elle conduit à faire des interactions entre Allemagne nazie et URSS stalinienne un élément essentiel de compréhension de leurs dynamiques, conduisant à la violence la plus extrême, à une brutalisation sans limites durant la Seconde Guerre mondiale.

13 Sans doute cette approche ne conduit-elle pas à une conceptualisation commune. Le lecteur circule dans la multiplicité des comparaisons mises en œuvre, avec un effet d'éclatement incontournable. Ceci montre peut-être les limites de l'exercice qui ne converge pas vers une unicité de la réflexion, dans un livre qui n'offre pas de conclusion générale, mais laisse à chacun le soin de relier les conclusions de chacun des chapitres. 
14 Par ailleurs, ces deux histoires, ainsi mises en comparaison, sont isolées du reste du contexte européen ; et ces confrontations terme à terme en laissent d'autres de côté, qui auraient peut-être permis de mieux évaluer la cohérence de chacune d'elles. Que cela concerne les modes de gouvernance (intégration ou non à l'État, place de la biopolitique) ou bien d'autres aspects évoqués dans l'ouvrage (p. ex. les formes de brutalisation mises en perspective avec celles du premier conflit mondial ou de la guerre civile en URSS), il est certain que des liens multiples apparaissent qui auraient pu être enrichis par des références à des expériences non autoritaires de la même époque.

\section{NOTES}

1. Henry Rousso, éd., Stalinisme et nazisme: Histoire et mémoire comparées, P.-Bruxelles : IHTPCNRS/Complexe, coll. « Histoire du temps présent », 1999.

2. Ian Kershaw et Moshe Lewin, eds., Stalinism and Nazism: Dictatorships in Comparison, Cambridge, MA : Cambridge University Press, 1997. 